\title{
PRESENCIA EN REDES SOCIALES Y ALTMÉTRICAS DE LOS PRINCIPALES AUTORES DE LA REVISTA EL PROFESIONAL DE LA INFORMACIÓN
}

\section{Daniel Torres-Salinas y Yusnelkis Milanés-Guisado}

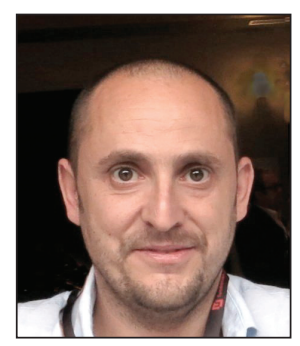

Daniel Torres-Salinas es doctor en documentación por la Universidad de Granada. Trabaja como bibliómetra en la Universidad de Navarra, donde analiza la producción y el impacto de su investigación. Es también investigador en el Grupo EC3 de la Universidad de Granada y desarrollador de herramientas de evaluación como Científica o los rankings I-UGR Rankings de Universidades según Campos y Disciplinas Científicas. Entre sus últimos proyectos destaca la creación junto a sus colegas de EC3 de la spin-off EC3metrics.

http://orcid.org/0000-0001-8790-3314

Universidad de Navarra, Centro de Investigación Médica Aplicada 31008 Pamplona, España torressalinas@gmail.com

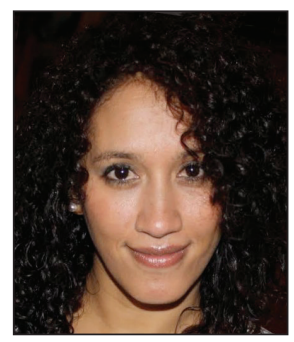

Yusnelkis Milanés-Guisado es licenciada y máster en bibliotecología y ciencias de la información. Es candidato a doctor por la Universidad de Granada. Ha trabajado en proyectos europeos y latinoamericanos de evaluación de la ciencia y gestión de la I+D+i. Sus actuales líneas de investigación son la evaluación del impacto de la investigación, bibliometría, análisis de patentes y la gestión de la I+D+i.

http://orcid.org/0000-0001-5461-9309

Universidad de Granada, Escuela Internacional de Posgrado Paz, 10. 18071 Granada, España ymilanes@gmail.com

\section{Resumen}

Se analiza una muestra discreta de los autores que más han publicado en la revista El profesional de la información (EPI) durante el período 2009-2013. Se estudia su presencia en las redes sociales y el impacto de sus publicaciones indexadas en la Web of Science a través de un grupo de altmétricas. Los resultados muestran que el $77 \%$ tienen perfil público en Google Scholar Profiles y el $70 \%$ tanto en LinkedIn como Mendeley. Esta plataforma se ha mostrado como la herramienta con estadísticas más elevadas; en comparación con las citas Web of Science obtiene el mayor valor de correlación (0,516). El 55\% tiene una cuenta en Twitter. Slideshare tiene más baja presencia (47\%), pero las presentaciones de los autores reciben mayor cantidad de vistas. Asimismo en el estudio se presentan altmétricas detalladas para 47 autores de EPI. Se finaliza sobre la dificultad que implica el análisis de las altmétricas a nivel de autor.

\section{Palabras clave}

Altmetrics, Métricas a nivel de autor, Web social, Redes sociales, Impacto, Documentación, Comunicación, El profesional de la información, EPI, Web of Science, Mendeley, CiteULike, Twitter, Slideshare.

Title: Presence on social networks and altmetrics of authors frequently published in the journal El profesional de la información

\begin{abstract}
We analyzed a discrete sample of the most frequent contributors to the journal El professional information (EPI) for the period 2009-2013. We studied the presence of these authors in social networks and the impact of their EPI publications indexed in Web of Science using different altmetrics. The results showed that $77 \%$ had a public profile on Google Scholar Profiles and $70 \%$ had an account on both Linkedln and Mendeley. This platform has served as the tool with higher statistics and obtained the highest correlation with the number of citations in Web of Science (0.516). Other networks were Twitter (55\%) and Slideshare (47\%); despite its lower presence in the sample, Slideshare offers users higher visibility, receiving the highest number of user hits. We also present detailed altmetrics for 47 EPI authors, ending with a reflection on the difficulty of analysing altmetrics at author level.
\end{abstract}

\section{Keywords}

Altmetrics, Author level metrics, Social web, Social networks, Library and information science, Communication, El profesional de la información, EPI, Web of Science, Mendeley, CiteULike, Twitter, Slideshare. 
Torres-Salinas, Daniel; Milanés-Guisado, Yusnelkis (2014). "Presencia en redes sociales y altmétricas de los principales autores de la revista El profesional de la información". El profesional de la información, julio-agosto, v. 23, n. 4, pp. 367372.

http://dx.doi.org/10.3145/epi.2014.jul.04

\section{Introducción}

Las altmétricas son consideradas una forma alternativa de medir el impacto de los resultados académicos de la investigación a través de la red social y están destinadas a mejorar y complementar las formas más tradicionales de evaluación mediante citas (Priem et al., 2010). Tienen en cuenta la difusión y visibilidad de la investigación en plataformas sociales, desde la búsqueda social de bibliografía a través de Facebook, a la discusión de resultados vía Twitter e incluyendo cualquier impacto que una publicación o autor pueda tener en la web social (Bar-Ilan et al., 2012).

Por ello ofrecen evidencias del alcance social de las publicaciones. Aunque sus potencialidades, validez, propiedades y estandarización (Wouters; Costas, 2012) no están aún del todo claras (Torres-Salinas; Cabezas-Clavijo, 2012; Eysenbach, 2011), abren la posibilidad de rastrear el impacto de los investigadores más allá de las citas, permitiendo a los investigadores conocer en menor tiempo la repercusión de sus trabajos.

Las altmétricas permiten a los investigadores conocer en menor tiempo la repercusión de sus trabajos

Una de sus principales fortalezas es proporcionar indicadores a nivel de artículo (Neylon; Wu, 2009; Costas; Zahedi; Wouters, 2014). El Article level metrics (ALM) es el nivel de análisis más conocido y utilizado (Tananbaum, 2013; Yan; Gerstein, 2011; Chamberlain, 2013), lo que permite valorar la repercusión de un artículo en entornos distintos a la revista donde ha sido publicado (Torres-salinas; Cabezas-Clavijo; Jiménez-Contreras, 2012). Otros trabajos han estudiado su uso en niveles de agregación como revistas (Nielsen, 2007; Cabezas-Clavijo; Torres-Salinas, 2012), universidades (Orduña-Malea; Ontalba-Ruipérez, 2013) y en menor medida autores.

En análisis altmétrico a nivel de autor, limitándonos al ámbito de la documentación, contamos con dos estudios: Barllan et al. (2012) analizan una muestra de 57 autores de la conferencia Science technology indicators en Leiden en 2010, recogiendo información sobre sus publicaciones y su repercusión en la web 2.0. Asimismo Haustein et al. (2014), con la misma muestra, amplían este estudio para analizar las relaciones de los investigadores en varias redes sociales.

Tomando como referente metodológico estos trabajos, en el presente artículo se revisan las altmétricas de un conjunto de autores en documentación a nivel de España. Ante la dificultad para analizar la comunidad completa se estudió una muestra discreta de los más productivos en El profe- sional de la información durante 2009-2013. Los objetivos específicos son los siguientes:

1. Analizar la presencia y utilización de redes sociales y perfiles científicos, a fin de comprobar si éstos son tenidos en cuenta por nuestra comunidad científica y su grado de penetración.

2. Conocer el impacto de los autores y los trabajos considerándose diferentes altmétricas calculadas para las publicaciones indexadas en la Web of Science en los últimos cinco años.

En definitiva tratamos de ofrecer una primera aproximación a la visibilidad web 2.0 de la producción científica de los autores en documentación que sirva como punta de lanza para estudios más exhaustivos.

\section{Material y métodos}

Se consideraron los autores con mayor número de artículos publicados en El profesional de la información durante el período 2009-2013. Se identificaron un total de 248 autores con un artículo o más. Se seleccionaron los autores con un umbral de 3 o más publicaciones en esta revista. Finalmente quedó una muestra de 47 autores. A continuación se realizó un trabajo manual en la web, para lo cual se revisaron sus respectivos perfiles en las siguientes redes sociales: Google Scholar Profiles, LinkedIn, Mendeley, Slideshare y Twitter. A partir de ellos se comprobó si los investigadores tenían perfiles abiertos en dichas plataformas y, en algunos casos, como en Twitter y Slideshare, se comprobó el número de seguidores.

A continuación se procedió a la descarga y análisis de las altmétricas de las publicaciones indexadas en Web of Science (WoS) entre 2009-2013. Se identificaron inicialmente un total de 626 publicaciones, incluyendo 184 duplicados debido a la co-autoría. La recopilación se realizó durante la segunda quincena de febrero y primera de marzo de 2014. La recuperación en WoS permitió identificar los DOIs en la mayoría de las publicaciones, y en los casos en los que no lo tenían, se procedió a buscarlos en otras fuentes como Crossref, Mendeley o Pubmed. Las publicaciones que finalmente no se identificaron fueron eliminadas quedando una muestra final de 426, una vez que también se suprimieron los duplicados.

Las altmétricas de estas publicaciones se recuperaron a través de la web de Impactstory que permite calcular métricas de Mendeley, Wikipedia, Twitter, Facebook, blogs y otras usando DOls, PubMed ID, usuario en Slideshare u otros identificadores. No obstante, con el fin de mejorar la calidad de los datos, en el caso de Mendeley se procedió a verificar los datos obtenidos a través de un trabajo manual en su propia interfaz. En el caso de Twitter se utilizó Topsy. En muchos casos se comprobó que las métricas aportadas por las distintas plataformas coincidían; pero en otros, como por 
ejemplo Twitter, se identificaron diferencias entre Impactstory y Topsy. En estos casos se procedió a asignar el valor más. El uso manual de la interfaz de Mendeley también permitió recuperar métricas en 34 publicaciones en las que no se tenían los DOls.

Las diferencias e inconsistencias entre los distintos proveedores de datos de Altmetrics es una cuestión que aún afecta a este tipo de estudios. Trabajos recientes como el de Zahedi, Fenner y Costas (2014), intentan esbozar este problema. Un paso en este sentido ha sido la reciente fusión de Impactstory con Altmetrics.Com, unión que no se había materializado en el momento de recogida de los datos del presente estudio. Esta fusión implica que se podrá tener acceso a nuevas métricas que Impactstory no proporcionaba antes como G+, Reddit o Facebook. Sin embargo, se pierde la cobertura de Twitter en muchos artículos y otros productos de investigación sin DOls, a los cuales se podía acceder con Topsy a través de los urls.

\section{Los resultados certifican que los autores más activos en EPI muestran también una clara orientación al mundo de la web 2.0}

Los artículos que no reciben ningún tweet se han contabilizado como 0 y no como valor nulo. Se ha escogido este procedimiento al considerar que el hecho de que una publicación no sea mencionada del todo puede ser considerado como 0 , ya que no tiene altmétrica (como cuando una publicación no es citada en lo absoluto y obtiene una puntuación de citas igual a 0). Un procedimiento similar es utilizado en Costas, Zahedi y Wouters (2014).

Finalmente los indicadores calculados a nivel global y para cada investigador fueron los siguientes: número tweets, media tweets por trabajo WoS, número lecturas, media lecturas por trabajo WoS, número bookmarks, media bookmarks por trabajo WoS, seguidores y vistas de Slides.

\section{Resultados}

La búsqueda de los autores arrojó una primera imagen sobre su presencia en las redes sociales y plataformas. Google Scholar Profiles, LinkedIn y Mendeley son las plataformas con mayor presencia. Google Scholar es la más utilizada: un $77 \%$ de los autores tienen un perfil público activo; Mendeley y Linkedln, ambas con un $70 \%$, la secundan (figura 1). El resultado es similar al encontrado por Barllan et al. (2012), según los cuales, 40 de los autores (70\%) analizados tenía cuenta

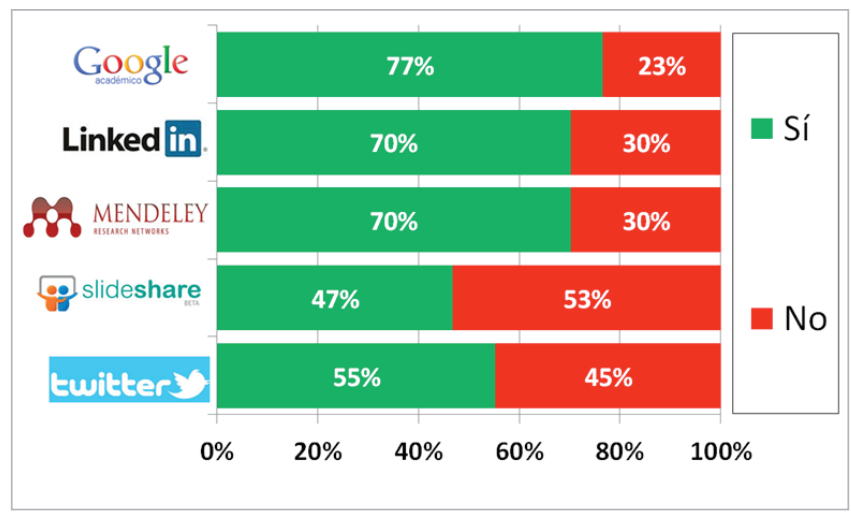

Figura 1. Presencia de los investigadores más productivos de EPI en diferentes redes sociales

en Linkedln, por lo que parece ser uno de los servicios de social media más populares. En Mendeley por su parte, también un $70 \%$ de los autores tienen perfiles aunque en su mayoría con poca actividad. Se consideraron perfiles con poca actividad o inactivos a aquellos creados pero con sus respectivos CVs sin completar, sin actualizaciones, cero o muy pocos resultados de investigación compartidos, cero membresía en grupos, etc. En Twitter el porcentaje es del 55\%. Bar-Ilan et al. (2012), identificaron que el $43 \%$ de los investigadores de su muestra tenían cuenta activa y Priem, Costello y Dzuba (2012), estimaron que sólo lo tiene un 2,5\% de académicos de 5 representativas universidades del Reino Unido y Estados Unidos. Finalmente Slideshare es la plataforma con menos presencia (47\%).

Tabla 1. Altmétricas de las publicaciones Web of Science (2009-2013) de los investigadores más productivos de EPI y tasa de correlación entre las métricas

\begin{tabular}{|c|c|c|c|c|}
\hline \multicolumn{5}{|c|}{ 1.A. Altmétricas de las publicaciones Web of Science } \\
\hline \multicolumn{3}{|c|}{ Indicador } & \multirow{2}{*}{$\begin{array}{l}\text { Valor } \\
644,00\end{array}$} & $\begin{array}{l}\text { Desviación } \\
\text { estándar }\end{array}$ \\
\hline \multirow{3}{*}{ Twitter } & \multicolumn{2}{|l|}{ Total tweets global } & & -- \\
\hline & \multicolumn{2}{|c|}{ Media tweets por trabajo WoS } & 1,55 & 4,44 \\
\hline & \multicolumn{2}{|c|}{ Total tweets promedio por autor } & 20,40 & 42,06 \\
\hline \multirow{3}{*}{ Mendeley } & \multicolumn{2}{|c|}{ Total lecturas global } & $5.212,00$ & -- \\
\hline & \multicolumn{2}{|c|}{ Media lecturas por trabajo WoS } & 12,43 & 16,19 \\
\hline & \multicolumn{2}{|c|}{ Total lecturas promedio por autor } & 144,36 & 313,68 \\
\hline \multirow{3}{*}{ CiteULike } & \multicolumn{2}{|c|}{ Total bookmarks global } & 370,00 & -- \\
\hline & \multicolumn{2}{|c|}{ Total bookmarks por trabajo WoS } & 0,88 & 2,78 \\
\hline & \multicolumn{2}{|c|}{$\begin{array}{l}\text { N. total bookmarks Promedio por } \\
\text { autor }\end{array}$} & 12,19 & 23,80 \\
\hline \multicolumn{5}{|c|}{ 1.B. Altmétricas en Slideshare } \\
\hline \multirow{3}{*}{ Slideshare } & \multicolumn{2}{|l|}{ Total vistas global } & 1.497 .697 & --- \\
\hline & \multicolumn{2}{|c|}{ Media vistas por presentación } & 3.213 & 5.094 \\
\hline & \multicolumn{2}{|c|}{ Total vistas promedio por autor } & 76.825 & 90.961 \\
\hline \multicolumn{5}{|c|}{ 1.C. Correlación de los diferentes indicadores para los 426 Web of Science analizados } \\
\hline & Twets & Lectore & Boo & Citas \\
\hline Twets & 1,000 & & & \\
\hline Lectores & $-0,062$ & 1,000 & & \\
\hline Bookmarks & 0,007 & 0,386 & & \\
\hline Citas & $-0,092$ & 0,516 & & 1,000 \\
\hline
\end{tabular}


En la tabla 1 se presentan las estadísticas generales de las altmétricas recopiladas. El mayor valor se obtiene en las métricas de Mendeley donde los 426 trabajos analizados alcanzan hasta un total de 5.212 lecturas, valor que lo sitúa muy lejos del resto de plataformas analizadas ya que las publicaciones recibieron 644 tweets y 370 bookmarks en CiteULike. Este patrón, donde Mendeley predomina, se repite para los otros indicadores calculados. Hay que mencionar cómo otros trabajos evidenciaron las bajas tasas de correlación entre los indicadores. Si comparamos las altmetrics con las citas Web of Science, el coeficiente más alto se obtiene con Mendeley donde se alcanza un valor de 0,51 muy similar al obtenido en otros estudios (Li; Thelwall; Giustini, 2011), que fue de 0,55. En relación a Slideshare hay que mencionar que pese a su baja utilización (figura 1) entre la comunidad, las presentaciones alcanzan valores muy altos de vistas. Así el conjunto de autores analizados suma hasta 1.497.697 vis- tas. La media de vistas por presentación se situó en 3.213. Estos números podrían indicar las potencialidades de esta plataforma para generar tráfico a sus usuarios.

En la tabla 2 se presentan las métricas agregadas a nivel de autor. La casi totalidad de los autores son académicos de universidades españolas. En cuanto a presencia en las redes sociales, 14 investigadores tienen cuenta abierta en todas las redes sociales analizadas, y tan sólo 2 no tienen ninguna presencia en la web social. Destacan por el número de seguidores en Twitter Antonio Fumero-Reverón (4.033), Javier Guallar (3.784) y José-Antonio Merlo-Vega (3.554). En Slideshare el mayor número de seguidores lo tienen Antonio Fumero-Reverón (630), Julio Alonso-Arévalo (600) y Daniel Torres-Salinas (270). El ranking de vistas globales del conjunto de presentaciones lo encabeza Daniel Torres-Salinas (314.488), seguido de Julio Alonso-Arévalo (247.336) y Jesús Tramullas (223.123).

Tabla 2. Presencia en redes sociales y altmetrics de las publicaciones Web of Science (2009-2013) de los investigadores más productivos de EPI

\begin{tabular}{|c|c|c|c|c|c|c|c|c|c|c|c|c|c|c|c|c|}
\hline & \multicolumn{8}{|c|}{ PRESENCIA EN REDES SOCIALES } & \multicolumn{8}{|c|}{ ALTMETRICS PARA TRABAJOS WEB OF SCIENCE (WOS) } \\
\hline INVESTIGADOR Y CENTRO & 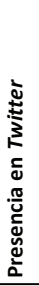 & 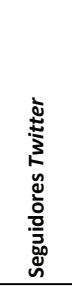 & 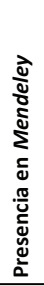 & 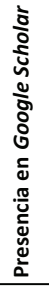 & 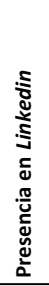 & 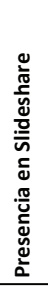 & 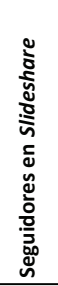 & 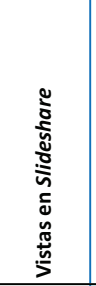 & & 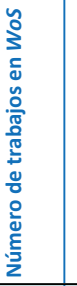 & 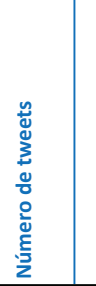 & 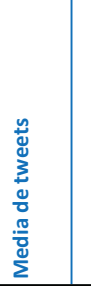 & 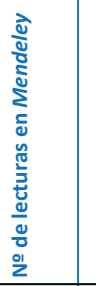 & 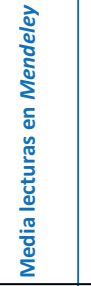 & 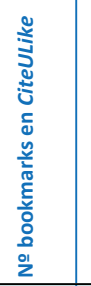 & 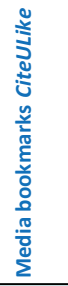 \\
\hline ABADAL-FALGUERAS, ERNEST UB & v & 186 & $\sqrt{ }$ & $\mathrm{v}$ & $\mathrm{V}$ & $\sqrt{ }$ & 137 & 19509 & & 15 & 18 & $1,3 \mid$ & 189 & 9 & 4 & 0,2 \\
\hline ALEIXANDRE-BENAVENT, RAFAEL UV & $\bigcirc$ & $\bigcirc$ & $\bigcirc$ & $v$ & $\checkmark$ & $\bigcirc$ & $\bigcirc$ & $\bigcirc$ & & 47 & 8 & 0,3 & 200 & 3,2 & 4 & 0,1 \\
\hline ALONSO-AREVALO, JULIO USAL & $\checkmark$ & 2.107 & $\mathrm{v}$ & $v$ & 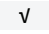 & $v$ & 600 & 247.336 & & 5 & $3 \mid$ & $0,5 \|$ & 221 & 74 & 58 & 19 \\
\hline ARROYO-VAZQUEZ, NATALIA FGSR & $v$ & 2.930 & $v$ & $v$ & $v$ & $v$ & 196 & 150.041 & & 3 & 0 & 0 & 28 & 9,3 & 0 & 0 \\
\hline CABEZAS-CLAVIJO, ALVARO UGR & v & 1.154 & $\checkmark$ & $v$ & $v$ & $v$ & 172 & 88.882 & & 10 & 110 & $11 \|$ & $168 \mid$ & 7,3 & $14 \|$ & 0,6 \\
\hline CODINA-BONILLA, LLUIS UPF & $v$ & 456 & $v$ & $v$ & $v$ & $v$ & 163 & 42.282 & & 14 & 0 & $0 \mid$ & 114 & 6,7 & 2 & 0,1 \\
\hline CORDON-GARCIA, JOSE-ANTONIO USAL & $v$ & 780 & $v$ & $v$ & $\bigcirc$ & $v$ & 174 & 41.071 & & 6 & 3 & $0,5 \|$ & 246 & 82 & 58 & 19 \\
\hline DELGADO-LOPEZ-COZAR, EMILIO UGR & $\bigcirc$ & $\bigcirc$ & $\bigcirc$ & $v$ & $\bigcirc$ & $v$ & 20 & 36.415 & & 23 & $48 \square$ & $2,7 \|$ & $301 \mid$ & 3,4 & 49 & 0,6 \\
\hline DE-MOYA-ANEGON, FELIX CCHS-CSIC & v & 622 & $v$ & $v$ & $v$ & $\bigcirc$ & $\bigcirc$ & $\bigcirc$ & & 52 & 101 & 2,1 & $750 \|$ & 2,9 & 69 & 0,3 \\
\hline DIAZ-NOCI, JAVIER UPF & $\mathrm{v}$ & 83 & $\mathrm{v}$ & $\mathrm{v}$ & 0 & $\mathrm{v}$ & 32 & 302 & & $7 \|$ & $4 \|$ & $0,6 \|$ & $85 \mid$ & 4,3 & 0 & 0 \\
\hline FEIJOO-GONZALEZ, CLAUDIO UPM & $\bigcirc$ & O & $v$ & O & $v$ & $\bigcirc$ & $\bigcirc$ & $\bigcirc$ & & 5 & 4 & $0,2 \|$ & 144 & 18 & 10 & 1,3 \\
\hline FERRER-SAPENA, ANTONIA UPV & O & O & $\mathrm{v}$ & $\mathrm{v}$ & 0 & 0 & 0 & 0 & & 9 & $24 \square$ & $3 \|$ & 124 || & 11 & 2 & 0,2 \\
\hline FUMERO-REVERON, ANTONIO UPM & $v$ & 4.033 & $\mathrm{v}$ & $\mathrm{v}$ & $\mathrm{v}$ & $\mathrm{v}$ & 630 & 8.498 & & 3 & 0 & 0 & 6\| & 2 & $2 \|$ & 0,7 \\
\hline GARCIA-MARCO, FRANCISCO-JAVIER UNIZAR & 0 & 0 & $v$ & $\mathrm{v}$ & $\mathrm{v}$ & 0 & 0 & 0 & & 5 & $3 \|$ & $0,6 \|$ & 59 & $30 \|$ & 4 | & 2 \\
\hline GIMENEZ-TOLEDO, ELEA CCHS-CSIC & $\bigcirc$ & $\bigcirc$ & $\bigcirc$ & $v$ & $v$ & $\bigcirc$ & $\bigcirc$ & $\bigcirc$ & & $16 \|$ & $8 \mid$ & $0,7 \mid$ & 53 & 1,8 & 3 & 0,1 \\
\hline GOMEZ-BARROSO, JOSE-LUIS UNED & 0 & 0 & $v$ & 0 & 0 & 0 & 0 & 0 & & $27 \mid$ & 4 & $0,2 \|$ & $159 \|$ & 3,1 | & 10 & 0,2 \\
\hline GONZALES-AGUILAR, AUDILIO UPVM3 & $\mathrm{v}$ & 56 & $\mathrm{v}$ & $v$ & $v$ & 0 & 0 & 0 & & 3 & 0 & 0 & $8 \|$ & 2,7 & $2 \mid$ & 0,7 \\
\hline GONZALEZ-PACANOWSKI, TONI Prestigia & $v$ & 232 & $v$ & $\bigcirc$ & $\checkmark$ & $v$ & 40 & --- & & 3 & 0 & 0 & 25 & 25 & 4 & 4 \\
\hline GUALLAR-DELGADO, JAVIER UB & $v$ & 3.784 & v & $v$ & $v$ & $v$ & 110 & 52.289 & & $11 \mid$ & $8 \mid$ & 0,9 & 56 & 2,2 & 0 & 0 \\
\hline HIDALGO-NUCHERA, ANTONIO UPM & O & $\bigcirc$ & $v$ & $v$ & $\bigcirc$ & $\bigcirc$ & $\bigcirc$ & $\bigcirc$ & & 9 & 0 & $0 \mid$ & 70 & 35 & 5 & 2,5 \\
\hline JIMENEZ-CONTRERAS, EVARISTO UGR & O & $\bigcirc$ & $\checkmark$ & $v$ & $\bigcirc$ & $\bigcirc$ & $\bigcirc$ & $\bigcirc$ & & 23 & 53 & $2,5 \|$ & 203 & $2,5 \|$ & 11 & 0,1 \\
\hline LEYDESDORFF, LOET UVA & v & 150 & $v$ & $v$ & v & $\bigcirc$ & $\bigcirc$ & $\bigcirc$ & & 114 & 161 & 1,5 L & 2056 & 1,6 & 120 & 0,1 \\
\hline MARCOS, MARI-CARMEN UPF & $v$ & 1.010 & 0 & $\mathrm{v}$ & $\mathrm{v}$ & $\mathrm{v}$ & --- & 1.907 & & 9 & 0 & 0 & 38 & 13 & 0 & 0 \\
\hline MARCOS-RECIO, JUAN-CARLOS UCM & $\checkmark$ & 115 & $\bigcirc$ & $\bigcirc$ & $v$ & $\bigcirc$ & $\bigcirc$ & $\bigcirc$ & & 7 & 0 & 0 & $19 \mid$ & 3,8 & $4 \|$ & 0,8 \\
\hline MARTINEZ-MENDEZ, FRANCISCO-JAVIER UM & $\bigcirc$ & $\bigcirc$ & $\bigcirc$ & v & $\bigcirc$ & $\bigcirc$ & $\bigcirc$ & $\bigcirc$ & & 4 & 0 & 0 & 40[ & $40 \mid$ & 3 & 3 \\
\hline MERLO-VEGA, JOSE-ANTONIO USAL & $\mathrm{v}$ & 3.554 & $\mathrm{v}$ & $\mathrm{v}$ & $\mathrm{v}$ & $v$ & 58 & 35.276 & & 3 & 0 & 0 & 33 & $33 \|$ & 5 & 5 \\
\hline MICO-SANZ, JOSEP-LLUIS URLL & 0 & 0 & 0 & 0 & 0 & 0 & 0 & 0 & & 4 & 0 & 0 & $31 \|$ & 5,2 & 0 & 0 \\
\hline MOREIRO, JOSE-ANTONIO UCBM & $\bigcirc$ & $\bigcirc$ & O & $v$ & $\bigcirc$ & $\bigcirc$ & --- & $\bigcirc$ & & 6 & $4 \|$ & $0,8 \|$ & 63 & 32 & 8 & 4 \\
\hline OLIVERA-ZALDUA, MARIA UCM & O & $\bigcirc$ & O & $\bigcirc$ & $\checkmark$ & $\bigcirc$ & O & $\bigcirc$ & & 7 & 0 & 0 & 25 & 13 & $4 \|$ & 2 \\
\hline ORDUNA-MALEA, ENRIQUE UPV & v & 251 & v & v & $v$ & $\bigcirc$ & --- & $\bigcirc$ & | & 11 & $38 \square$ & $3,5 \|$ & $56 \|$ & 6,2 & 2 & 0,2 \\
\hline PASTOR-SANCHEZ, JUAN-ANTONIO UM & O & $\bigcirc$ & $\checkmark$ & $\checkmark$ & $v$ & 0 & O & $\bigcirc$ & & 6 & 0 & 0 & 24 & 24 & $1 \|$ & 1 \\
\hline PEDRAZA-JIMENEZ, RAFAEL UPF & v & 76 & $v$ & $v$ & $v$ & $v$ & 50 & 73.456 & & 8 & 0 & 0 & $74 \|$ & 3,9 & 2 & 0,1 \\
\hline PEREZ-MONTORO, MARIO UB & $v$ & 75 & $v$ & O & $v$ & $v$ & 95 & --- & & 6 & 14 & 2,3 & 56 & 14 & 3 & 0,8 \\
\hline PESET-MANCEBO, FERNANDA UPV & $\bigcirc$ & $\bigcirc$ & $v$ & v & $\checkmark$ & $v$ & 27 & --- & & 11 & 0 & $0 \|$ & $47 \mid$ & $4,7 \mid$ & 5 & 0,5 \\
\hline RAMIREZ-POSADA, MARIA-VICTORIA UPVM3 & $\bigcirc$ & $\bigcirc$ & $\bigcirc$ & $\bigcirc$ & O & $\bigcirc$ & $\bigcirc$ & $\bigcirc$ & & 4 & 0 & 0 & 8 & 2 & 2 & 0,5 \\
\hline ROBINSON-GARCIA, NICOLAS UGR & $v$ & 456 & $v$ & $v$ & $\checkmark$ & $v$ & 105 & 72.071 & & 15 & 160 & $11 \mid$ & $97 \mid$ & 4,4 & 1 & 0,1 \\
\hline RODRIGUEZ-GAIRIN, JOSEP-MANUEL UB & $\bigcirc$ & 0 & $v$ & $\mathrm{v}$ & $v$ & $x$ & $\bigcirc$ & $\bigcirc$ & & 6 & $4 \|$ & $0,7 \|$ & 56 & 56 & $2 \mid$ & 2 \\
\hline RODRIGUEZ-PARADA, CONCEPCION UB & $\bigcirc$ & $\bigcirc$ & $\bigcirc$ & $\bigcirc$ & $\bigcirc$ & $\bigcirc$ & $\bigcirc$ & $\bigcirc$ & & 4 & 0 & 0 & $21 \mid$ & 5,3 & $2 \mid$ & 0,5 \\
\hline ROVIRA-FONTANALS, CRISTOFOL IUPF & $\mathrm{v}$ & 576 & $\mathrm{v}$ & $\mathrm{v}$ & $\mathrm{v}$ & $\mathrm{v}$ & 6 & 13.711 & & 6 & 0 & 0 & $37 \mid$ & 5,3 & 1 & 0,1 \\
\hline RUIZ-PEREZ, RAFAEL UGR & $\bigcirc$ & $\bigcirc$ & $\bigcirc$ & $v$ & $\bigcirc$ & $\bigcirc$ & $\bigcirc$ & $\bigcirc$ & & 12 & $8 \|$ & $1 \mid$ & $66 \|$ & $2,2 \|$ & 15 & 0,5 \\
\hline SANCHEZ-CUADRADO, SONIA UC3M & $\bigcirc$ & $\bigcirc$ & $v$ & $v$ & $\checkmark$ & $\bigcirc$ & $\bigcirc$ & $\bigcirc$ & & 9 & $11 \|$ & $1,6 \|$ & 48 & $16 \|$ & 5 & 1,7 \\
\hline SANCHEZ-VIGIL, JUAN-MIGUEL CUM & $\checkmark$ & 19 & $v$ & $\bigcirc$ & $\checkmark$ & $\bigcirc$ & $\bigcirc$ & $\bigcirc$ & & 9 & 0 & 0 & $21 \mid$ & 4,2 & $4 \|$ & 0,8 \\
\hline SAORIN-PEREZ, TOMAS UM & $\mathrm{v}$ & 84 & $v$ & $\mathrm{v}$ & $\mathrm{v}$ & $\mathrm{v}$ & 44 & 16.202 & & 4 & 0 & 0 & $12 \|$ & 3 & 0 & 0 \\
\hline SERRANO-COBOS, JORGE UPV & $v$ & 1.170 & 0 & $\mathrm{v}$ & $v$ & $\mathrm{v}$ & 230 & 22.821 & & 6 & $2 \mid$ & $0,3 \|$ & $59 \|$ & 6,6 & $4 \|$ & 0,4 \\
\hline TORRES-SALINAS, DANIEL UN & $\mathrm{v}$ & 1.001 & $\mathrm{v}$ & $\mathrm{v}$ & $\mathrm{v}$ & $\mathrm{v}$ & 270 & 314.488 & & 33 & $137 \square$ & 4,4 & 464|| & 4,1 & $60 \|$ & 0,5 \\
\hline TRAMULLAS-SAZ, JESUS UNIZAR & $v$ & 1.656 & $\mathrm{v}$ & $v$ & $\mathrm{v}$ & $v$ & 147 & 223.123 & & $8 \|$ & $11 \|$ & 1,6 | & 46 & 12 & $2 \mid$ & 0,5 \\
\hline XIFRA-TRIADU, JORDI UPF & $\bigcirc$ & $\bigcirc$ & 0 & $\mathrm{v}$ & 0 & 0 & $\bigcirc$ & $\bigcirc$ & $\amalg$ & 18 & $10 \|$ & 0,6 & $79 \mid$ & $5,3 \|$ & $7 \|$ & 0,5 \\
\hline
\end{tabular}


En las altmétricas agregadas de las publicaciones Web of Science de los autores y en relación al número de tweets, presentan los mejores valores Loet Leydesdorff (161), Nicolás Robinson-García (161) y Daniel Torres-Salinas (137). En Mendeley los valores máximos de lecturas lo alcanza Loet Leydesdorff (2.056), Félix De-Moya-Anegón (750) y Daniel Torres-Salinas (464). Si consideramos CiteUlike se repite el mismo ranking que en Mendeley, aunque con valores mucho menores. El hecho de que estos autores tengan la mayor cantidad de publicaciones, podría influir en el comportamiento de los valores del número de lecturas en estas plataformas.

\section{Consideraciones finales}

Se ha presentado un análisis de las altmétricas tanto de trabajos como de autores relevantes en el ámbito de la documentación española. Si bien es cierto que la muestra es limitada y quizás no extrapolable a toda la comunidad, ha permitido verificar la amplia penetración de las redes sociales en los investigadores más activos en El profesional de la información. Ahora mismo Google Scholar Profile es la plataforma más empleada, por el $77 \%$ de la muestra, y Slideshare la menos utilizada, con sólo el $47 \%$. Sin embargo Slideshare se revela como una de las plataformas que mayor tráfico genera a los usuarios: los 22 investigadores que tienen cuenta en esta red consiguen un promedio de 3.213 vistas por presentación. Una cifra que contrasta con los resultados del resto de productos analizados. Asimismo el análisis nos ha permitido establecer el impacto de 426 trabajos donde Mendeley se ejerce como la herramienta más fiable al ofrecernos las estadísticas más elevadas, los trabajos WoS tienen una media de 12,43 lecturas.

Se confirman las dificultades que implica el cálculo de altmétricas a nivel de artículo: detección de afiliaciones, identificación de alias con autores, falta de estandarización en los títulos, etc.

Si comparamos los datos de la presencia de investigadores en las redes sociales analizadas con los resultados obtenidos en Haustein et al. (2014), los autores analizados presentan valores muy equiparables en el caso de LinkedIn y Twitter pero muy divergentes para Mendeley y Google Scholar Profiles. En estos dos últimos casos los autores de El profesional de la información parecen hacer un mayor uso de estas redes, si bien hay que tener en cuenta que los resultados de Haustein fueron recopilados en 2012 y los nuestros en 2014. En cualquier caso los resultados certifican que los autores más activos en EPI muestran también una clara orientación al mundo de la web 2.0.

Finalmente se confirman las dificultades que implica el cálculo de altmétricas a nivel de artículo ya apuntadas en otros trabajos (Torres-Salinas; Cabezas-Clavijo; Jiménez-Contreras, 2013) (detección de afiliaciones, identificación de nicks con autores, falta de estandarización en los títulos, etc.) que complican y ralentizan el cálculo de indicadores frente a los
Tabla 3. Comparativa de la presencia en redes sociales de los investigadores según dos estudios diferentes

\begin{tabular}{|l|c|c|}
\cline { 2 - 3 } \multicolumn{1}{c|}{} & $\begin{array}{c}\text { Haustein et al., } \\
\mathbf{2 0 1 4}\end{array}$ & $\begin{array}{c}\text { Estudio } \\
\text { actual }\end{array}$ \\
\hline Red social & \multicolumn{2}{c|}{$\begin{array}{c}\text { \% Investigadores } \\
\text { con cuenta }\end{array}$} \\
\hline Linkedln & 67 & 70 \\
\hline Twitter & 43 & 55 \\
\hline Mendeley & 23 & 70 \\
\hline Google Scholar Profiles & 22 & 77 \\
\hline
\end{tabular}

indicadores más clásicos. Sin embargo, pese a la pérdida de información que puede suponer la búsqueda de indicadores a través de altmétricas, se han podido calcular éstas para 47 investigadores. Los resultados obtenidos han permitido identificar investigadores y profesionales ya destacados previamente en nuestra comunidad bien por su producción científica e impacto internacional (por ejemplo Loet Leydesdorff o De-Moya-Anegón) o bien por su gran visibilidad profesional (Julio Alonso-Arévalo).

Slideshare se revela como una de las plataformas que mayor tráfico genera; los 22 investigadores que tienen cuenta en esta red consiguen un promedio 3.213 vistas por presentación

En general, el análisis de los individuos es complicado incluso con los indicadores bibliométricos más tradicionales. Una de las limitaciones importantes de este estudio, sino la mayor, es el tamaño de su muestra y su especificidad. En altmetría el trabajo con grupos de autores exige un trabajo adicional ya que, como sucede en bibliometría, la normalización de los nombres y afiliaciones de los autores es uno de los principales problemas. Añadir además la propia identificación de los perfiles de los investigadores en las redes sociales y su normalización, incrementa esta dificultad. Se ha confirmado que el trabajo con grupos de autores reducidos y con cierto conocimiento de la rama de conocimiento y su comunidad científica, puede facilitar el trabajo, pero siempre demanda un esfuerzo añadido. A pesar de las limitaciones anteriores, y otras como la diversidad de medios sociales y plataformas proveedoras de datos de altmetría, incluso la falta aún de comprensión de por qué se usan algunos de estos medios como Mendeley, el estudio puede ser merecedor de un cierto valor metodológico. Futuros estudios, ampliando considerablemente la muestra, las redes sociales y los indicadores, deberían corroborar o al menos permitir la discusión de los resultados presentados aquí.

\section{Bibliografía}

Bar-Ilan, Judit; Haustein, Stefanie; Peters, Isabella; Priem, Jason; Shema, Hadas; Terliesner, Jens (2012). "Beyond citations: Scholars' visibility on the social web". En: $17^{\text {th }}$ Int l conf 
on science and technology indicators, Montreal, 5-8 Sept. http://arxiv.org/abs/1205.5611

Cabezas-Clavijo, Álvaro; Torres-Salinas, Daniel (2010). “Indicadores de uso y participación en las revistas científicas 2.0: el caso de PLoS one". El profesional de la información, v. 19, n. 4, pp. 431-434.

http://eprints.rclis.org/14801/1/431-434.pdf

http://dx.doi.org/10.3145/epi.2010.jul.14

Chamberlain, Scott (2013). "Consuming article-level metrics: observations and lessons". Information standards quarterly, v. 25, n. 2, pp. 4-13.

http://www.niso.org/apps/group_public/download. php/11303/FE_Chamberlain_Consuming_ALMs_isq_ v25no2.pdf

http://dx.doi.org/10.3789/isqv25no2.2013.02

Costas, Rodrigo; Zahedi, Zohreh; Wouters, Paul (2014). Do 'altmetrics' correlate with citations? Extensive comparison of altmetric indicators with citations from a multidisciplinary perspective.

http://arxiv.org/abs/1401.4321

Eysenbach, Gunther (2011). "Can tweets predict citations? Metrics of social impact based on Twitter and correlation with traditional metrics of scientific impact". Journal of medical internet research, v. 13, n. 4, p. 123.

http://dx.doi.org/10.2196/jmir.2012

Haustein, Stefanie; Peters, Isabella; Bar-Ilan, Judit; Priem, Jason; Shema, Hadas; Terliesner, Jens (2014). “Coverage and adoption of altmetrics sources in the bibliometric community". Scientometrics, On Line First.

http://arxiv.org/abs/1304.7300

http://dx.doi.org/10.1007/s11192-013-1221-3

Li, Xuemei; Thelwall, Mike; Giustini, Dean (2011). "Validating online reference managers for scholarly impact measurement". Scientometrics, v. 91, n. 2, pp. 461-471. http://dx.doi.org/10.1007/s11192-011-0580-x

Neylon, Cameron; Wu, Shirley (2009). "Article-level metrics and the evolution of scientific impact". PLoS biol, v. 7, n. 11, e1000242. http://dx.doi.org/10.1371/journal.pbio.1000242

Nielsen, Finn (2007). "Scientific citations in Wikipedia". First Monday, v. 12, pp. 8-6. http://firstmonday.org/article/view/1997/1872

Orduña-Malea, Enrique; Ontalba-Ruipérez, José-Antonio (2013). "Selective linking from social platforms to university websites: a case study of the Spanish academic system". Scientometrics, v. 95, n. 2, pp. 593-614.

http://arxiv.org/pdf/1310.5815.pdf

http://dx.doi.org/10.1007/s11192-012-0851-1

Priem, Jason; Costello, Kaitlin; Dzuba, Tyler (2012). “Prevalence and use of Twitter among scholars". En: Symposium on informetric and scientometric research. New Orleans, LA, USA, October 12.

http://dx.doi.org/10.6084/m9.figshare.104629

Priem, Jason; Taraborelli, Dario; Groth, Paul; Neylon, Cameron (2010). Alt-metrics: a manifesto.

http://altmetrics.org/manifesto

Tananbaum, Greg (2013). Article level metrics. A Sparc primer. http://sparc.arl.org/sites/default/files/sparc-alm-primer.pdf

Torres-Salinas, Daniel; Cabezas-Clavijo, Álvaro (2013). “Altmetrics: no todo lo que se puede contar, cuenta". Anuario Thinkepi, v. 7, pp. 114-117.

Torres-Salinas, Daniel; Cabezas-Clavijo, Álvaro; JiménezContreras, Evaristo (2013). "Altmetrics: nuevos indicadores para la comunicación científica en la web 2.0". Comunicar, v. XXI, n. 41, pp. 56-60

http://dx.doi.org/10.3916/C41-2013-05

Wouters, Paul; Costas, Rodrigo (2012). Users, narcissism and control: tracking the impact of scholarly publications in the $21^{\text {st }}$ century.

http://research-acumen.eu/wp-content/uploads/Usersnarcissism-and-control.pdf

Yan, Koon-Kiu; Gerstein, Mark (2011). "The spread of scientific information: insights from the web usage statistics in PLoS article-level metrics". PLoS one, v. 6, n. 5. http://dx.doi.org/10.1371/journal.pone.0019917

Zahedi, Zohreh; Fenner, Martin; Costas, Rodrigo (2014). How consistent are altmetrics providers? Study of 1000 PLoS one publications using the PLOS ALM, Mendeley and Altmetric.com APIs.

http://dx.doi.org/10.6084/m9.figshare.1041821

\section{Suscripción EPI sólo online}

Pensando sobre todo en los posibles suscriptores latinoamericanos, ya no es obligatorio pagar la suscripción impresa de EPI para acceder a la online.

EPI se ofrece a instituciones en suscripción "sólo online" a un precio considerablemente más reducido $(101,65+21 \%$ IVA euros/año = 123 euros/año), puesto que en esta modalidad no hay que cubrir los gastos de imprenta ni de correo postal.

Para los suscriptores particulares, el precio de la sucripción "sólo online" es de $62+21 \%$ IVA euros/año $=75$ euros/año. 